Vittorio Gallese

\title{
Embodied Simulation. Its Bearing on Aesthetic Experience and the Dialogue Between Neuroscience and the Humanities
}

\section{Introduction}

The artist is he who arrests the spectacle in which most men take part without really seeing it and who makes it visible to the most 'human' among them.

(Merleau-Ponty, 1945/1964, p. 18)

Is there something invisible about reality that art reveals to all human beings? What is the 'invisible' Merleau-Ponty is talking about? 'Già non attendere' io tua dimanda, s'io mintuassi, come tu t'inmii. This is what Dante wrote in the IX Canto of Paradiso, addressing the blessed soul of Folco da Marsiglia. In these splendid verses that bear witness to the wonderful linguistic creativity of great poetry, we can find an illuminating anticipation of the notion of empathy. To empathize means understanding the other from within, as also suggested by the German term for empathy - Einfühlung, to feel inside, which interestingly enough was first used within the debate on aesthetics. This 'intuition' implies for the ego the possibility of connecting to the You without losing itself in it, attributing to the other actions, emotions and sensations that, however, what the ego knows as part of its own vital experience.

Empathizing means understanding what the other is doing and experiencing, without necessarily feeling compassion or being motivated to help. These latter characteristics refer to the notion of sympathy, which is often confused with that of empathy. Empathy is experiencing a renewed interest in psychology and aesthetics, thanks to the revaluation of the body, greatly promoted by recent discoveries of cognitive neuroscience, such as that of mirror neurons.

The present paper deals with the encounter with the other, both when it takes place in real life as well as when it is mediated by what the other has created, like when relating to works of art. It is proposed that such an encounter - in both instances - does not decline exclusively in linguistic and abstract terms, but encompasses a grounding bodily nature. 
I introduce embodied simulation and discuss its potential heuristics in social cognition and, most importantly, in the dialogue with the humanities. This perspective, which emphasizes the sensory-motor nature of human cognition, offers many advantages: 1) it allows to frame human cognition within an evolutionary scenario, which privileges exaptation over adaptation, continuity over discontinuity and challenging 'deus-ex-machina-like' solutions, like abrupt genetic mutations (i.e., the 'syntax gene'); 2) it enables a comparative perspective, connecting human cognitive skills to their evolutionary antecedents in non-human primates and 3) it enables a new take on aesthetic experience, linking the production of cultural artefacts to their reception.

In the present essay, I approach from a neuroscientific perspective human social relation and the production and reception of human cultural artefacts connecting them to their underlying neural mechanisms. I submit that any form of aesthetic experience induced by human relations to cultural artefacts is a mediated form of intersubjectivity, where the cultural artefact connects the subjectivity of the producer of the artefacts with that of their recipients. Before addressing the aesthetic experience of fictional world, let us have a look at what neuroscience revealed about the relation between action, perception and cognition, shedding new light on the bodily nature of intersubjectivity and its underlying neural mechanisms.

\section{Embodied Simulation and Intersubjectivity as Intercorporeality}

It is fair to say that neuroscience with its recent discoveries has literally revolutionized how to conceive perception and imagination and, more generally, has revealed the tight relation between action, perception and cognition. Cognitive abilities, like the mapping of space and its perception, the perception of objects occupying our visual landscape, the hierarchical representation of action with respect to a distal outcome, and the detection of motor goals and action anticipation, are all possible because of the motor system and its peculiar functional architecture, organized in terms of goal-directed motor acts (see Gallese, 2000, 2009, 2014, 2016, 2018a; Gallese et al. 2009; Gallese \& Cuccio, 2015). As it will be clearer in the second part of this article, the proper development of such functional architecture likely scaffolds more cognitively sophisticated social cognitive abilities, like the production/reception of cultural artefacts and fictional world.

The discovery of mirror neurons in the brain of macaques, neurons that are active both during the execution of actions and their observation when performed by others (Gallese et al. 1996; Rizzolatti et al. 1996), and the subsequent discovery of mirror mechanisms in the human brain (see Gallese et al. 2004; Gallese, 2007, 2014, 2018a), revealed that a direct access to the meaning of others' behaviour is 
available, an access that is different from the explicit attribution of propositional attitudes. The appreciation of purpose in others' behaviour does not exclusively rely on explicit propositional inference, because motor outcomes and motor intentions are part of the 'vocabulary' spoken by the motor system (Rizzolatti, Fogassi \& Gallese, 2000). In many circumstances, we do not explicitly ascribe intentions to others; we simply detect them by means of motor simulation, that is, by activating part of motor system without moving.

The discovery of the mirror mechanism for actions in humans led to the hypothesis that mirror neurons could be just the tip of a much bigger iceberg, because a similar functional mechanism could in principle also apply to the domain of emotions and sensations (Goldman \& Gallese, 2000; Gallese, 2003). The empirical evidence obtained in the following years confirmed this hypothesis. Other mirroring mechanisms are involved in our ability to share the emotions and sensations of others: the very same nervous structures involved in the subjective experience of emotions and sensations are also active when such emotions and sensations are recognized in others (for a review, see Gallese \& Guerra, 2015; Gallese \& Cuccio, 2015). Witnessing someone expressing a given emotion (e.g., disgust) or undergoing a given sensation (e.g., touch, pain) recruits some of the visceromotor (e.g., anterior insula) and sensory-motor (e.g., SII, ventral premotor cortex) brain areas activated when one experiences the same emotion or sensation, respectively. Other cortical regions are exclusively recruited for one's own and not for others' emotions or are activated for one's own tactile sensation but are actually deactivated when observing someone else being touched (for review, see Gallese, 2014, 2018a; Gallese \& Cuccio, 2015). I proposed to qualify all these mirroring mechanisms as the expression of the same functional mechanism: embodied simulation.

Embodied simulation theory uses a notion of embodiment according to which mental states or processes are embodied because of their bodily format (Gallese, 2003, 2005, 2014). The bodily format of a mental representation constrains what such mental representation can represent because of the bodily constraints posed by the specific nature of the human body. Similar constraints apply both to the representations of one's own actions, emotions or sensations and to those of others. Hence, embodied simulation is the reuse of mental states and processes involving representations that have a bodily format. Sensory-motor systems, originally evolved to guide our interactions with the world, once decoupled from the common final motor pathway and dynamically reconnected with other cortical areas can be put into the service of newly acquired cognitive skills, like understanding others. The experience of our actions, emotions and sensations and of those of others always takes place within a we-centric dimension (see Gallese, 2001, 2003). 
The activation of embodied simulation is the recall of the background bodily knowledge we acquire during our factual relation to the world of inanimate objects and other sentient beings. We recruit this knowledge in several different situations, such as when mapping events within our peripersonal space; when confronting with manipulable objects; when witnessing others' actions, emotions and sensory experiences; when remembering past experiences; when planning future actions, when engaging in fictional experiences and when comprehending linguistic descriptions of facts, actions, and events (see Gallese \& Cuccio, 2015). Indeed, also when we read or listen to narratives, we literally embody them by activating part of our sensory-motor system. The activation of motor representations in the brain of the reader or listener has been demonstrated at the phono-articulatory level, as well as during the processing of action-related linguistic expressions (words and sentences) and morpho-syntactic aspects of language (for review, see Pulvermüller, 2005; Gallese, 2008; Glenberg \& Gallese 2012). This evidence points to a causal role of embodied simulation in language processing and understanding, thus opening up new interesting research scenarios to be also applied to the reception of fictional narrative (Wojciehowski \& Gallese, 2011).

\section{Embodied Simulation and Imagination}

What about imagination? Mirror mechanisms are just one instantiation of embodied simulation: when mirror mechanisms are activated, the simulation process is triggered by perception, like when observing someone performing an action, expressing an emotion, or undergoing a somatosensory stimulation. However, embodied simulation can also occur when we imagine perceiving something or imagine doing something. When we imagine a visual scene, we activate the same cortical visual areas normally active when we do perceive the same visual scene. Similarly, mental motor imagery and real action both activate a common network of cortical and subcortical motor centres, such as the primary motor cortex, the premotor cortex, the supplementary motor area, the basal ganglia and the cerebellum (for review, see Wojciehowski \& Gallese, 2011). A recent high-density electroencephalography (EEG) study showed that the brain circuits that inhibit action execution are partly the same as those that allow us to imagine to act (Angelini et al., 2015).

Thus, visual and motor mental imagery are not exclusively symbolic and propositional. They both rely on and depend upon the activation of sensory-motor brain regions. Visual imagery is somehow equivalent to simulating an actual visual experience, and motor imagery is somehow equivalent to simulating an actual motor experience. In other words, visual and motor imagery do qualify as further forms of embodied simulation. When indulging in visual or mental motor 
imagery, we reuse our visual or motor neural apparatus to imagine things and situations we are not actually perceiving or doing (Gallese, 2011).

Indeed, when viewed from a neuroscientific perspective, the border separating real and imaginary worlds appears much less sharp and clear than what humans thought for centuries. The similarity between our responses to real and fictional events transpires even at the level of single neurons. A recent study by Caggiano et al. (2011) showed that macaques' mirror neurons respond to both the observation of real actions performed by the experimenter physically present in front of them and their filmed footage displayed on a computer screen. Thus, the neurobiological mechanisms enabling the connection to the 'real world' largely overlap with those connected to fictional world.

As it will become evident in the next section, this aspect is particularly interesting when referred to cultural artefacts and their reception. Hence, embodied simulation theory can be used to both account for how we perceive the world and how we imagine it and build a world of fiction and experience it. My hypothesis is that the world of cultural artefacts is 'felt' not too differently from how we feel the more prosaic world we encounter in daily life. We feel for and empathize with fictional images and characters in ways that are similar to how we feel for our real social partners, although with qualifying differences (see Gallese, 2011, 2017, 2018b).

\section{Embodied Simulation and Aesthetics: Visual Art}

What is the relevance of neuroscience to aesthetics and aesthetic experience? Cognitive neuroscience is currently empirically investigating art and aesthetics with different approaches to address a number of different issues and questions: 1) to use artistic expressions to understand how the brain works, 2) to localize in the brain -and/or reduce to its functioning-aesthetic concepts like beauty or the sublime, 3) to study the brain to explain art, 4) to study the brain-body in relation to artistic expressions and 5) to understand the constitutive elements of aesthetic experience and the genesis of aesthetic concepts. As previously argued, neuroscience can maximize its heuristic power, particularly if adopting the last approach, enabling to deconstruct and explain the genesis of aesthetic concepts from a bottom-up perspective (Gallese 2017, 2018b; Gallese \& Guerra, 2015).

In the second half of the XIX century, Hildebrand (1893) maintained that when beholding (artistic) images, the perception of the spatiality of the image is the result of a constructive sensory-motor process. In Hildebrand's view, space is a product and not an a priori experience as Kant suggests: the reality of the artistic image lies in its effectual nature, conceived as both the result of the causes that produced it and the effect that it produces in the observer. 
According to this constructivist logic, the value of a work of art lies in the ability to establish a relationship between the artist's intention and the observer's reconstruction of it, thus establishing a direct relationship between the creation of the object and the artistic pleasure it produces. Hildebrand proposed that knowing the object is equivalent to knowing the process by which it has been created. Another of Hildebrand's ideas is even more in line with the hypothesis put forward here: our experience of observed images has fundamental connotations in motor terms (see Freedberg \& Gallese, 2007; Gallese, 2011, 2012, 2017; Gallese \& Guerra, 2015). This has indeed been confirmed by a series of experiments carried out in our laboratory: the observation of letters of the Roman alphabet, Chinese ideograms or meaningless scribbles, all written by hand, activates the beholders' motor representation of their hand (Heiman, Umiltà \& Gallese, 2013). In two other studies, we demonstrated that a similar motor simulation of hand gestures is evoked when looking at the cuts on canvas by Lucio Fontana (Umiltà et al., 2012) or at the dynamic brushstrokes on canvas by Franz Kline (Sbriscia-Fioretti et al., 2013).

The visible traces of the creative gestures activate in the observer the specific motor areas controlling the execution of the same gestures. Beholders' eyes catch not only provides information about the shape, direction and texture of the cuts or strokes but by means of embodied simulation, they breach into the actual motor expression of the artist when creating the artwork. The sensory-motor component of image perception, together with the jointly evoked sensory and emotional reactions, allows beholders to feel the artwork in an embodied manner (Gallese, 2017, 2018b).

What we see is not the 'simple visual' recording in our brain of what stands in front of our eyes but the result of a complex construction, whose outcome is the result of the fundamental contribution of our body with its motor potentialities, our senses and emotions, our imagination and our memories. We must definitely abandon the outdated concept of solipsistic and 'purely visual' vision. Vision is a complex experience, intrinsically synaesthetic, that is made of attributes that largely exceed the mere transposition in visual coordinates of what we experience any time we lay our eyes on something. The expression 'laying the eyes' indeed betrays the haptic quality of vision: our eyes are not just optical instruments but also a 'hand' touching and exploring the visible, turning it into something seen by someone (Gallese, 2018b, p. 77).

So far, I dealt with the role of bodily engagement in the aesthetic experience of artistic images. What about the explicit aesthetic judgement of beauty? We recently explored whether beholders' sensory-motor engagement with the emotional content of works of art contributes to the formation of their objective aesthetic judgement of beauty (Ardizzi et al., 2018). To this purpose, participants' 
sensory-motor engagement was modulated by asking them to overtly contract the corrugator supercilii facial muscles or to refrain from any voluntary facial movement, while judging the aesthetic value of painful and neutral facial expressions in selected examples of Renaissance and Baroque paintings. Results demonstrated a specific increase in the explicit rating of aesthetic beauty of paintings showing painful facial expressions during the congruent activation of the corrugator supercilii muscles. The same manipulation of participants' facial expression did not affect their aesthetic judgements of beauty of neutral facial expressions. Furthermore, participants' empathetic traits and expertise in art were found to correlate directly with the amplitude of the motor enactment effect on aesthetic judgements. These results suggest that the mimetic stance towards the beholden images affects not only the empathic engagement they evoke but also the explicit aesthetic judgements of their artistic beauty. As we wrote in this research article,

\begin{abstract}
'The present results demonstrate for the first time that beholders' sensorymotor engagement with the bodily based painful experiences portrayed in works of art represents a consistent bottom-up mechanism that significantly plays a role in the formation of an objective aesthetic evaluation of beauty and that interacts with top-down factors (i.e., expertise in art, empathetic traits). Despite the emphasis of the original empathy theorists on the role played in aesthetic evaluation by bodily based resonance with the artwork, the neuroaesthetic tradition seems to have mainly inherited the "Kantian" view of the aesthetic stance, that is, a detached state in which the experience of beauty is separated from beholders' bodily involvement. Indeed, sensory-motor mechanisms are not comprised in the major models of aesthetic appreciation. [...] The present results reveal the necessity of extending the role of sensory-motor engagement beyond the domain of artist's gestures to the implicit resonance of such engagement with the emotional content of works of art.' (Ardizzi et al., 2018, p. 8).
\end{abstract}

\title{
5. Embodied Simulation and Aesthetics: Film Immersion
}

How does cinema work? How can something so patently false be at the same time powerful enough to elicit our full immersion with its contents and to evoke our identification with the characters and situations narrated on the screen, triggering emotions sometimes even more powerful than those we experience in real life? How and at which level does the movie engage the viewer? Kracauer proposed that the moving image engages the viewer "physiologically before he is in a position to respond intellectually". Moreover, it elicits 'a "resonance effect" provoking in the audience such kinaesthetic responses as muscular reflexes, motor impulses, or the like. In any case, objective movement acts as a physiological stimulus'. (Kracauer, 1997). 
We started addressing these questions experimentally by focussing on two aspects of film style: camera movements and montage. As David Bordwell wrote, camera movements are a 'persuasive surrogate for our subjective movement through an objective space' (Bordwell, 1977, p. 23). We performed a combined behavioural and high-density EEG study investigating motor cortex activation during observation of videos showing an actor performing hand actions, like grasping an object placed on a table (Heimann et al., 2014). Stimuli were filmed using the camera in four different ways: filming from a fixed position; zooming in on the scene; approaching the scene by means of a dolly, that is, moving the camera along tracks, and approaching the scene by means of a steadicam, a camera secured to the body of a moving camera man so to minimize vibrations and oscillations. Results demonstrated a consistently stronger motor activation of participants' brain during the observation of videos that were filmed while approaching the scene with the Steadicam. The EEG results were paralleled by the behavioural ones: when asked about how they experienced the different videos, participants judged those filmed with the Steadicam as those most resembling the visual experience of approaching the scene by walking towards it.

The results of Heimann et al. (2014), however, raised further questions regarding the precise nature of the motor simulation at stake. As a first possibility, they could indicate that the Steadicam added ecological validity to the presentations, leading to a stronger activation of the mirror mechanism in response to the observation of the hand actions executed by the actor filmed in the scene. An alternative or additional explanation might refer to the simulation of the movement of the cameraman walking towards the scene while filming it. In order to clarify this issue, we recently ran another experiment where we filmed an empty room with a still camera, with zoom and Steadicam. Results showed a stronger activation of spectators' motor cortex during observation of videos filmed with the Steadicam, thus demonstrating for the first time that filmic means such as camera movements alone can modulate spectators' bodily engagement with film (Heimann et al., 2019).

Another important element of film style is editing: thanks to editing, we are able to lose ourselves in the film's narrative fiction, happily unaware of the artificiality of what we are seeing. Every film is a concatenation of images, and for the most part, they are linked together by continuity editing, which permits the conjunction of frames and sequences that can be very different in their visual contents. The sequence of a hand grasping a set of keys can be followed by that of a person entering a house. A hand seizing a revolver can be followed immediately by the sequence of a body collapsing onto the floor, hit by a bullet from the self-same revolver from the previous sequence. Despite the fact that the perception of the flow is created by a succession of discontinuous images, the editing 
guarantees space-temporal and causal unity between the sequences (Gallese \& Guerra, 2015).

Various empirical studies have shown that during the viewing of a film, blinking and saccadic eye movements tend to take place when the viewer's attention is at a low ebb, such as during the interruption between two events. If the editing cut happens at this point, the viewer notices it less. The efficacy of the continuity editing technique depends greatly on the typology of the images prior to and following the cut. The masking of the cut is successful when the sequence that comes immediately after the cut is congruent with the expectations generated by that which preceded it. One of the rules that governs continuous editing is closely related to the relationship between the predictive anticipation of what we will see and the continuous perception of the narrated events: this rule, which concerns the position of the camera vis-à-vis the actors being filmed, is called the ' $180^{\circ}$ rule'; it provides that the space in which the action is being filmed is divided into two by an imaginary line, the axis. The camera is placed in one half, while the other half is the so-called 'pro-filmic space' in which the action is filmed.

Previous studies have shown that editing adhering to the $180^{\circ}$ rule does not generate a sensation of discontinuity, while editing that violates the rule does. Sequences containing camera movements adhering to the $180^{\circ}$ rule are sufficiently similar to visual perception in real life, which is constantly interrupted by blinking and saccadic eye movements, and so they pass unobserved. If the changes in the position of the camera taking the shot following the cut are sufficiently small, they become 'transparent' and therefore invisible to the spectator. Owing to its dramatic breach of our usual perceptual visual modalities, violation of the $180^{\circ}$ rule, known in cinematic terms as 'crossing the line', does not permit such transparency.

We investigated how the brain of spectators responds to sequences respecting or violating the $180^{\circ}$ rule: although both conditions show perceptual discontinuities between the frames shot before and after the cut (given that the camera was moved), only the frames edited crossing the line arouse the viewer's notice. According to our hypothesis, this cannot entirely depend upon purely visualattentional effects. One of the consequences of crossing the line is that the frame shot before the cut is completely inverted and specular in the second frame: what was on the right before the cut, after the cut is on the left and vice versa. The viewer has the sensation that his perspective has been inverted. Therefore, the second frame, which violates the $180^{\circ}$ rule, is not only profoundly disturbing from a perceptual point of view but also characterized by sensory-motor incongruence. This results in a temporary suspension of the embodied simulation through which the viewer becomes immersed in the scene, which means that his attention 
tends to focus more on the cut than on the content. The perceptual dissonance the viewer experiences while watching a scene edited crossing the line is caused by the violation of sensory-motor expectations generated by experience of corporeal interaction and the way things happen in the world.

We used high-density EEG to verify this hypothesis empirically, recording the neural basis of perception of the two types of editing (continuous and discontinuous editing). Cuts and edits in general elicit early brain responses, indicating the registration of syntactic violations, as known from language, music and action processing. However, continuity and discontinuity edits differ from each other regarding later brain responses, likely indicating the differences in spatial remapping as well as in the degree of conscious awareness of spectators' perception. The perceptual analysis of sequences assembled with discontinuity editing does not depend on visual attention but on how much these sequences deviate from our visual perception in real life, as the perspective from which we normally view the world is disoriented. Film editing stimulates the perceptual competences and their underlying neural mechanisms that are usually used in our visual interaction with the world around us: editing engages spectators' embodied visual perception that uses previous sensory-motor experiences from the real world to map, revise and detect the discontinuities of the moving images in a film.

Film experience and film immersion do not depend just on concepts and propositions, but rely on sensory-motor schemas, which get the viewer literally in touch with the screen, shaping a multimodal form of simulation, which exploits all the potentialities of our brain-body system.

\section{The Biocultural Paradigm and the Dialogue Between Neuroscience and the Humanities}

The previous sections provided a series of examples of the way neuroscience can today profitably address topics once considered of exclusive pertinence of the humanities. This approach is often criticized as the expression of an ill-founded form of reductionism and, to make things worse, as the epitome of undue interference in someone else's courtyard. However, as we have seen in section 4, neuroscience can address arts, aesthetics and, more generally, the world of fiction in different ways, asking different questions. Thus, it looks over reductive if not preposterous to treat these different approaches under the same monolithic tag.

Luckily enough, things are changing, as epitomized by the growing consensus upon what has been designated as the 'biocultural paradigm' (see Wojciehowski \& Gallese, 2011, 2018; Casadei, 2018; Cometa, 2018a,b; Gambino \& Pulvirenti, 2018; Gallese 2019). The biocultural paradigm posits that any human 
technology is at the same time the expression of the human mind and humans' bodily nature, as the latter scaffolds the former. Many scholars are questioning the rigid dichotomy separating culture from nature, arguing that culture - as an extension or outgrowth of nature - is the evolved capacity of human beings to develop and use instrumental intelligence (Gallese, 2019). Culture can be described as a naturally evolved type of human cognitive technology. As argued by the French philosopher Simondon (2001), technology exceeds any narrow utilitarian purpose: as technology expands, it produces new relations between people and things, between people and people, or between things and things. Technology is a network of relations: far from marking our alienation from the natural world, technology is what mediates between humankind and nature. This point has been recently reinstated by the cognitive archaeologist Ian Hodder (2012) with the notion of 'entanglement', that is, the different ways humans and things relate to one another.

I recently proposed that human cultural evolution can be conceived as a perpetually dynamic process of cognitive technological development, where the first prehistoric lithic tools and the iPhone 7 used to shoot feature movies, like the recent Unsane by Steven Soderberg (2018), represent two different and temporally distinct expressions of the same technological dimension (see Gallese, 2019). What we currently designate as 'cultural artefacts' or 'works of art' constitute no exception to this perspective. The biocultural paradigm posits that any human technology is at the same time the expression of the human mind and humans' bodily nature, as the latter scaffolds the former.

When we speak of nature and culture, we are always dealing with two sides of the same coin. Nevertheless, the human mind and humans' bodily nature should be kept separate at the operational level, as they are best approached and studied from multiple perspectives, according to multiple methodologies, leading to correspondingly different levels and languages of description. The real challenge for the biocultural paradigm is to define bridge concepts and/or empirically test how to deconstruct, revise or even dissolve many of the conceptual tools we currently use to discuss art, film, literature and their reception (Gallese, 2019).

Neuroscience can provide new concepts bridging nature and culture, as it is well equipped to address the conceptualization of human culture, art, film and literature by investigating the brain-body mechanisms underpinning both the creative process and the reception of its outcomes. By studying the brain-body in relation to artistic expressions and their reception, we can better understand the constitutive elements of aesthetic experience and the genesis of aesthetic concepts. Indeed, if one considers human culture and nature as mutually interacting domains, any biological description of human cognitive/cultural traits is neither 
forced to surrender to deterministic innatism nor forced to neglect historical and ethnological cultural diversity (Wojciehowski \& Gallese, 2011; Gallese, 2019).

Once neuroscience will have abandoned its delusion of explanatory selfsufficiency, it will greatly benefit from a transdisciplinary dialogue and collaboration with the humanities, which could bring to neuroscientists' attention an enormous corpus of knowledge testifying the great cultural diversity and at the same time some universal principle presiding over the way human beings relate to reality through the lens of art and fiction (Wojchehowski and Gallese, 2018; Gallese, 2019).

Mirror neurons and embodied simulation do not consist of stereotyped and undifferentiated responses. They are both context dependent and idiosyncratically linked to individuals' personal historical, social and biological identity. The political and ethical impacts of all cultural artefacts clearly depend upon how these modulating factors affect the bodily responses they trigger. The response to cultural artefacts is always characterized by a projective quality, related to how personal life history determines the development of embodied cognition, as the latter is the outcome of bodily habits and implicit memories (Gallese, 2011; Gallese \& Guerra, 2015). The scientific investigation of the mechanisms enabling the experience of cultural artefacts does not imply, let alone posit, cultural homogeneity: the same neurobiological mechanisms are likely at stake in every culture, as they are part of humans' biological fabric. However, we speak of different cultures or different responses to cultural artefacts within the same culture, just because these mechanisms are plastic and bear the mark of each individual's personal identity, thus leading to personalized forms of embodiment (Wojchehowski \& Gallese, 2018; Gallese, 2019).

\section{Acknowledgments}

This work was supported by Maria Paola Chiesi and the PRIN grant on Perception, Performativity and the Cognitive Sciences.

\section{Summary}

Embodied simulation, a basic functional mechanism of our brain, and its neural underpinnings are discussed and connected to intersubjectivity and the reception of human cultural artefacts, like visual arts and film. Embodied simulation provides a unified account of both non-verbal and verbal aspects of interpersonal relations that likely play an important role in shaping not only the self and his/her relation to others, but also shared cultural practices. Embodied simulation sheds new light on aesthetic experience and is proposed as a key element for the dialogue between neuroscience and the humanities within the biocultural paradigm.

Keywords: Aesthetic experience, biocultural paradigm, body, embodied simulation, film, immersion, mirror neurons, visual art. 


\section{Körperliche Nachahmung. Ihre Bedeutung für Ästhetische Erfahrungen und einen Dialog zwischen Neurowissenschaft und Humanwissenschaft.}

\section{Zusammenfassung}

Körperliche Nachahmung, ein grundlegender funktionaler Mechanismus unseres Gehirns, und ihre neuronalen Grundlagen werden in Verbindung mit Intersubjektvität und der Rezeption menschlicher kultureller Artefakte wie optische Künste und Film diskutiert. Körperliche Nachahmung ermöglicht eine einheitliche Beschreibung von sowohl non-verbalen als auch verbalen Aspekten interpersoneller Beziehungen, die wahrscheinlich eine wichtige Rolle in der Gestaltung nicht nur des Selbst und seiner Beziehung zu Anderen, sondern auch gemeinsamer kultureller Praktiken, spielt. Körperliche Nachahmung läßt ästhetische Erfahungen in neuem Licht erscheinen und wird als Schlüsselfaktor für den Dialog zwischen Neurowissenschaft und Humanwissenschaft innerhalb des bio-kulturellen Paradigmas vorgeschlagen.

Schlüsselwörter: Ästhetische Erfahrung, bio-kulturelles Paradigma, Körper, körperliche Nachahmung, Film, Immersion, Spiegelneuronen, optische Künste.

\section{References}

Angelini, M., Calbi, M., Ferrari, A., Sbriscia-Fioretti, B., Franca, M., Gallese, V., \& Umiltà, M.A. (2015). Motor inhibition during overt and covert actions: An electrical neuroimaging study. PLoS One 10(5). $\mathrm{e} 0126800$.

Ardizzi, M., Ferroni, F., Siri,F., Umiltà, M.A., Cotti, A., Calbi, M.,...Gallese, V. (2018). Beholders's sensorimotor engagement enhances aesthetic rating of pictorial facial expressions of pain. Psychological Research, Aug 3. [Epub ahead of print].

Bordwell, D. (1977), Camera movement and cinematic space, Ciné-Tracts, 2, 19-25.

Caggiano, V., Fogassi, L., Rizzolatti, G., Pomper, J. K., Thier, P., Giese, M.A., \& Casile, A. (2011). View-based encoding of actions in mirror neurons of area $\mathrm{f5}$ in macaque premotor cortex. Current Biology, 21(2), 144-8.

Casadei, A. (2018). Biologia della Letteratura. Corpo, Stile, Storia. Milano: Il Saggiatore.

Cometa, M. (2018a). Perché le storie ci aiutano a vivere. La letteratura Necessaria. Milano: Raffaello Cortina Editore.

Cometa, M. (2018b). Letteratura e darwinismo. Introduzione alla biopoetica. Roma: Carocci Editore.

Freedberg, D. \& Gallese, V. (2007). Motion, emotion and empathy in aesthetic experience. Trends in Cognitive Sciences, 11, 197-203.

Gallese, V. (2000). The inner sense of action: Agency and motor representations. Journal of Consciousness Studies, 7, 23-40.

Gallese, V. (2001). The "Shared Manifold” Hypothesis: from mirror neurons to empathy. Journal of Consciousness Studies, 8, $N^{\circ}$ 5-7, 33-50.

Gallese, V. (2003). The manifold nature of interpersonal relations: The quest for a common mechanism. Philosophical Transactions of the Royal Society of London B 358, 517-528.

Gallese, V. (2005). Embodied simulation: From neurons to phenomenal experience. Phenomenology and the Cognitive Sciences, 4, 23-48.

Gallese, V. (2007). Before and below theory of mind: Embodied simulation and the neural correlates of social cognition. Philosophical Transactions of the Royal Society of London B, 362, 659-669.

Gallese, V. (2008). Mirror neurons and the social nature of language: The neural exploitation hypothesis. Social Neuroscience, 3, 317-333.

Gallese, V. (2009). Motor abstraction: A neuroscientific account of how action goals and intentions are mapped and understood. Psychol Res, 73(4), 486-498.

Gallese, V. (2011) Embodied simulation theory: Imagination and memory. Neuropsychoanalysis, 13(2), 196-200. 


\section{GESTALT THEORY, Vol. 41, No.2}

Gallese, V. (2012). Aby Warburg and the dialogue among aesthetics, biology and physiology. Ph, 2, 48-62.

Gallese, V. (2014). Bodily selves in relation: Embodied simulation as second-person perspective on intersubjectivity. Philosophical Transactions of the Royal Society B, 369, 20130177, published 28 April 2014.

Gallese, V. (2016). Finding the body in the brain. From simulation theory to embodied simulation. In H. Kornblith \& B. McLaughlin (Ed.). Alvin Goldman and his Critics. (297-317). New York: Blackwell.

Gallese, V. (2017). Visions of the body: Embodied simulation and aesthetic experience. Aisthesis, 1(1), 41-50.

Gallese, V. (2018a). Embodied simulation and its role in cognition. Reti, Saperi, Linguaggi, in press.

Gallese, V. (2018b). The power of images: A view from the brain-body. Phenomenology and Mind, 14, 70-79.

Gallese, V. (2019). A bodily take on aesthetics. Performativity and embodied simulation. In

Gallese, V., Fadiga, L., Fogassi, L., \& Rizzolatti, G. (1996). Action recognition in the premotor cortex. Brain, $119,593-609$.

Gallese, V., Keysers, C., \& Rizzolatti, G., (2004). A unifying view of the basis of social cognition. Trends Cognitive Sciences, 8, 396-403.

Gallese, V., Rochat, M., Cossu, G., \& Sinigaglia, C. (2009). Motor cognition and its role in the phylogeny and ontogeny of action understanding. Developmental Psychology, 45, 103-113.

Gallese, V., \& Cuccio, V. (2015). The paradigmatic body. Embodied simulation, intersubjectivity and the bodily self. In T. Metzinger, \& J. M. Windt (Ed.). Open MIND. (1-23). Frankfurt: MIND Group.

Gallese, V., \& Guerra, M. (2015). Lo Schermo Empatico: Cinema e Neuroscienze. Milan: Raffaello Cortina Editore.

Gambino, R., \& Pulvirenti G. (2018). Storie Menti Mondi. Approccio Neuroermeneutico alla Lelleratura. Milano-Udine: Mimesis.

Glenberg, A., \& Gallese, V. (2012) Action-based language: A theory of language acquisition production and comprehension. Cortex, 48(7), 905-22.

Goldman, A., \& Gallese, V. (2000). Reply to Schulkin. Trends in Cognitive Sciences, 4, 255-256.

Heimann, K., Umiltà, M. A., \& Gallese V. (2013). How the motor-cortex distinguishes among letters, unknown symbols and scribbles. A high density EEG study. Neuropsychologia, 51, 2833-2840.

Heimann K., Umiltà M. A., Guerra M., \& Gallese V. (2014). Moving mirrors: A high density EEG study investigating the effects of camera movements on motor cortex activation during action observation. Journal of Cognitive Neuroscience, 26(9), 2087-2101.

Heimann, K., Calbi, M., Umiltà, M.A., Guerra, M., Fingerhut, J. \& Gallese, V. (2019). Embodying the camera: An EEG study on the effect of camera movements on film spectators' sensorimotor cortex activation. PloS ONE, in press.

Hildebrand, von A. (1893). Das Problem der Form in der Bildenden Kunst. Strasbourg: Heitz.

Hodder, I. (2012). Entangled: An Archaeology of the Relationships between Humans and Things. Oxford: Wiley Blackwell.

Kracauer, S. (1997). Theory of Film: The Redemption of Physical Reality. Princeton, NJ: Princetown University Press. Merleau-Ponty, M. (1945/1964). Sense and Non-Sense. (H. L. Dreyfus and P. Allen Dreyfus, Trans.). Evanston, IL: Northwestern University Press.

Pulvermüller, F. (2005). Brain mechanisms linking language and action. Nature Reviews Neuroscience, 6(7), 576-582.

Rizzolatti, G., Fogassi, L., \& Gallese, V. (2000). Cortical mechanisms subserving object grasping and action recognition: A new view on the cortical motor functions. In M. S. Gazzaniga (Ed.). The New Cognitive Neurosciences, 2nd Edition (539-552). Cambridge: A Bradford Book, MIT Press, Ma.

Rizzolatti, G., Fadiga, L., Gallese, V., \& Fogassi, L. (1996). Premotor cortex and the recognition of motor actions. Cognitive Brain Research, 3, 131-141.

Sbriscia-Fioretti, B., Berchio, C., Freedberg, D., Gallese, V., \& Umiltà, M. A. (2013). ERP modulation during observation of abstract paintings by Franz Kline. PloS ONE, 8(10), e75241.

Simondon, G. (2001). Du Mode d'Existence des Objets Techniques. Paris: Aubier.

Umiltà, M. A., Berchio, C., Sestito M., Freedberg, D., \& Gallese, V. (2012). Abstract art and cortical motor activation: An EEG study. Frontiers in Human Neuroscience, 6, 311.

Wojciehowski, H.C. \& Gallese, V. (2011). How stories make us feel. Toward an embodied narratology. California Italian Studies, Vol. 2, Issue 1.

Wojciehowski, H. C., \& Gallese, V. (2018). "Introduction.” Costellazioni: Rivista di lingue e letterature, 5, 9-22. 
Vittorio Gallese, b. 1959, MD and trained neurologist, is professor of Psychobiology at the University of Parma, Italy, and adjunct senior research scholar at the Department of Art History and Archeology, Columbia University, New York, USA. As a cognitive neuroscientist, his research focuses on the relation between the sensory-motor system and cognition by investigating the neurobiological and bodily grounding of intersubjectivity, empathy, language and aesthetics. He is the author of more than 200 scientific publications and two books. Address: Department of Medicine and Surgery, Unit of Neuroscience, University of Parma, Via Volturno, Fisiologia Umana Palazzina E, 43126 Parma, Italy.

Email: vittorio.gallese@unipr.it 